\title{
Compositional Model Checking for Linear-Time Temporal Logic
}

\author{
Roope Kaivola \\ University of Helsinki, Department of Computer Science \\ Teollisuuskatu 23, SF-00510 Helsinki, Finland \\ tel. +358-0-708 4163, fax. +358-0-708 4441 \\ email rkaivola@cc.helsinki.fi
}

\begin{abstract}
Temporal logic model checking is a useful method for verifying properties of finile-slate concurrent systems. However, due to the state explosion problem modular methods are essential in making the verification task manageable. One such method is to verify that certain properties $\phi_{i}$ are true of the submodules $M_{i}$ of the system in all environments, and that the required property $\phi$ is a logical implication of these. This paper presents an algorithm deciding whether a nexltime-less linear lemporal logic formula $\phi$ is true of a distributed variable module $M$ in all environments. There are two versions of the algorilhm: one allowing no fairness requirements and one for strongly fair concurrency. Both versions run in time $O\left(|M| \cdot 2^{c \cdot|\phi|}\right)$. In addition to presenting the algorithms it is shown that given some reasonable assumptions the method is complete in the sense that all formulas $\phi$ true of $M_{1} \| M_{2}$ can be verified by it.
\end{abstract}

\section{Introduction}

One of the most important approaches to practical verification of propositional temporal logic properties of finite-state programs is automated model-checking: the execution of a program is modelled by a finite graph which can be directly interpreted as a temporal logic model, and a model-checking algorithm for the appropriate temporal logic can be run on the model. For many propositional temporal logics the model checking algorithms are of relatively low time-complexity, e.g. linear in size of the model and singly exponential in the size of the formula for the standard linear temporal logic [LP85].

However, despite the low time-complexity of model checking, the size of the execution graph is still often a prohibitive factor. An essential reason for this stateexplosion problem is the modelling of concurrency by arbitrary interleavings of the atomic actions of the concurrent processes. In the general case the size of the complete model is exponential in the number of concurrent processes.

One way to avoid this problem is verifying the system directly on the basis of the individual processes without constructing a global execution graph. Here we follow the approach advocated by [MP91]. In this method a system $M$ consists of several concurrent modules $M_{1}\|\ldots\| M_{n}$. Verifying that $\phi$ is true of $M$ is done by finding lemmas $\phi_{1}, \ldots, \phi_{n}$ such that each $\phi_{i}$ holds of $M_{i}$ in every possible environment and $\phi_{1} \wedge \ldots \wedge \phi_{n} \Rightarrow \phi$ is a theorem.

In this paper we describe an algorithm deciding whether a nexttime-less temporal logic formula $\phi$ is true of a module $M$ in every possible environment. There are two versions of the algorithm: the basic case in which the concurrency is modelled by 
potentially unfair interleavings, and the enhanced case with fair concurrency. Both versions run in a time $O\left(|M| \cdot 2^{c \cdot|\phi|}\right)$. In other words, using this algorithm it costs no more to decide whether $\phi_{i}$ is true of $M_{i}$ in all (fair) environments than it costs in the standard linear temporal logic model checking [LP85] to decide whether $\phi_{i}$ is true of $M_{i}$ without any environment. The method of communication between processes is by distributed variables. These are shared variables with the restriction that only the process owning a variable may change its value.

We do not discuss the issue of how the lemmas $\phi_{i}$ are to be found, and leave it to the responsibility of the human verifier of a system. However, in Section 5 we prove that given certain reasonable assumptions the lemmas always exist. This means that the method is complete in the sense that every formula $\phi$ true of $M_{1} \| M_{2}$ is, at least in theory, verifiable by it.

The modularity issues discussed here have been addressed by several researchers. [BKP84], [Pnu85] and [Bar86] describe compositional linear temporal logic semantics for distributed-variable systems similar to ours. However, their point of interest lies more in the axiomatic semantics and compositional proof rules than in presenting an explicit algorithm for modular verification. A method for compositional modelchecking using $M C T L$, an extension of $C T L$ allowing restricted linear temporal logic formulas and fairness requirements as assumptions, is discussed in [Jos89]. A modular model checking algorithm for a logic tailored for Petri-nets is presented in [BE91]. It is unclear whether either of these methods can be applied to the model and the linear temporal logic used here. An approach to $C T L^{*}$ model checking in which the enviromment of a module is modelled by an additional interface module is presented in [CLM89]. This differs from our approach in the use of interface processes and in the underlying communication method. The problem of modular verification of communicating Moore-machines is addressed by [GL91]. However, it does not seem possible to transfer their results directly into an asynchronous distributed-variable model.

The paper proceeds as follows: we first recall some standard definitions and properties of nexttime-less linear temporal logic and present the concepts of a module and parallel composition of modules. Then we define the core concept of a satisfiability graph and show that in a sense it encodes all the necessary information about the behaviour of a module in any environment. To illustrate the approach in practice, a mutual exclusion protocol is verified as an example. In Section 5 the results are extended to deal with fair concurrency.

\section{Linear temporal logic}

In this section we recall the definitions of standard nexttime-less linear temporal logic.

Definition 2.1 The alphabet of propositional linear temporal logic $L T L^{\prime}$ consists of the set $A P$ of atomic propositions and of the symbols $(),, \neg, \vee, U$. The well-formed formulas (wffs) of $L T L^{\prime}$ are as follows:

- if $\phi \in A P$, then $\phi$ is a wf,

- if $\phi_{1}$ and $\phi_{2}$ are wffs, then $\left(\neg \phi_{1}\right),\left(\phi_{1} \vee \phi_{2}\right)$ and $\left(\phi_{1} U \phi_{2}\right)$ are wffs, and

- there are no other wffs.

We use the abbreviations $T \equiv_{d f}(p \vee(\neg p))$ for some fixed $p \in A P,\left(\phi_{1} \wedge \phi_{2}\right) \equiv_{d f}$ $\left(\neg\left(\left(\neg \phi_{1}\right) \vee\left(\neg \phi_{2}\right)\right)\right),\left(\phi_{1} \Rightarrow \phi_{2}\right) \equiv_{d f}\left(\left(\neg \phi_{1}\right) \vee \phi_{2}\right),(\diamond \phi) \equiv_{d f}(T \mathcal{U} \phi),(\square \phi) \equiv_{d f}$ 
$(\neg(\diamond(\neg \phi))),\left(\phi_{1} \mathcal{U}_{w} \phi_{2}\right) \equiv_{d}\left(\left(\phi_{1} \mathcal{U} \phi_{2}\right) \vee\left(\square \phi_{1}\right)\right)$, and the ordinary precedence rules to reduce the number of parentheses.

Definition 2.2 A truth set $v$ is a set of atomic propositions, $v \subseteq A P$. A truth set sequence $\sigma$ is a finite or infinite sequence of truth sets, $\sigma=\left(v_{1}, v_{2}, \ldots\right)$. If $\sigma$ is a truth set sequence, $\sigma_{n}$ is the $n:$ th element of $\sigma, \sigma^{(n)}$ is the truth set sequence obtained by leaving the first $n$ elements out of $\sigma$, and $\sigma \cap A$ is the truth set sequence $\left(v_{1} \cap A, v_{2} \cap A, \ldots\right)$.

Definition 2.3 A Kripke-model $K$ is an ordered 4-tuple $K=(S, I, R, V)$, where $S$ is the set of states, $I \subseteq S$ is the set of initial states, $R \subseteq S \times S$ is the transition relation, and $V: S \rightarrow P(A P)$ is a valuation expressing the atomic formulas true in a state. $(P(X)$ denotes the powerset of $X$.)

A palh $p$ in a Kripke-model $K$ is a finite or infinite sequence $p=\left(s_{1}, s_{2}, \ldots\right)$ such that $s_{1} \in I$, and each $\left(s_{i}, s_{i+1}\right) \in R$. A path $p$ is a fullpath iff either $p$ is infinite or there is no further state reachable from the last state of $p$, i.e. if $s_{n}$ is the last state of $p$ then for all $s,\left(s_{n}, s\right) \notin R$. The truth set sequence corresponding to a fullpath $p=\left(s_{1}, s_{2}, \ldots\right)$, denoted $V(p)$, is $\left(V\left(s_{1}\right), V\left(s_{2}\right), \ldots\right)$.

Next we augment Kripke-models with fairness constraints expressed in terms of transition sets [LP85]. The notion of fairness used is the so-called strong fairness, meaning that if an event is possible infinitely often it has to be realised infinitely often, too.

Definition 2.4 A fair Kriple-model $K_{f}$ is an ordered 5-tuple $K_{f}=(S, I, R, V, F)$, where $(S, I, R, V)$ is a Kripke-model and $F$ is a finite set of faimess sets $F=$ $\left\{f_{1}, \ldots f_{n}\right\}$ such that $\bigcup_{f_{i} \in F} f_{i}=R$.

The paths and fullpaths of $K_{f}$ are those of the Kripke-model $(S, I, R, V)$. A fairness set $f_{i} \in F$ is enabled in a state $s \in S$ iff there is an $s^{\prime}$ such that $\left(s, s^{\prime}\right) \in f_{i}$. A fairness set $f_{i}$ is active in a transition $\left(s, s^{\prime}\right)$ iff $\left(s, s^{\prime}\right) \in f_{i}$. A fullpath $p$ is fair iff every fairness set $f_{i}$ that is enabled in infinitely many states of $p$ is active in infinitely many transitions of $p$, too.

Definition 2.5 An $L T L^{\prime}$-formula $\phi$ is true in a truth set sequence $\sigma=\left(v_{1}, v_{2}, \ldots\right)$ i.e. $\sigma \vDash \phi$, according to the following rules:

- If $\phi \in A P$, then $\sigma \vDash \phi$ iff $\phi \in v_{1}$.

- $\sigma \vDash \neg \phi$ iff not $\sigma \vDash \phi$.

- $\sigma \vDash \phi_{1} \vee \phi_{2}$ iff $\sigma \vDash \phi_{1}$ or $\sigma \vDash \phi_{2}$.

- $\sigma \vDash \phi_{1} \mathcal{U} \phi_{2}$ iff $\exists: 0 \leq i<|\sigma|$, such that $\sigma^{(i)} \vDash \phi_{2}$ and for all $0 \leq j<i$, $\sigma^{(j)} \vDash \phi_{1}$.

If $\phi$ is true in every truth set sequence $\sigma$ we say that $\phi$ is a theorem and write $\models \phi$. An $L T L^{\prime}$-formula $\phi$ is true in a (fair) Kripke-model $K$, denoted $K \vDash \phi$, iff $V(p) \vDash \phi$ for every (fair) fullpath $p$ of $K$.

The operators have their conventional meanings. The reflexivity of $U$ and the lack of a nexttime-operator allow us to overlook truth sets in a truth set sequence if they do not differ from their predecessor.

Definition 2.6 Let $\sigma=\left(v_{1}, v_{2}, \ldots\right)$ be a truth set sequence. The reduced form of $\sigma$, denoted by $\operatorname{red}(\sigma)$ is constructed by removing from $\sigma$ all $v_{i}$ such that $v_{i}=v_{i-1}$. If $A \subseteq A P$ and $\operatorname{red}(\sigma \cap A)=\operatorname{red}\left(\sigma^{\prime} \cap A\right)$ we say that $\sigma$ and $\sigma^{\prime}$ are stuttering equivalent modulo $A$ and write $\sigma \approx \sigma^{\prime}(\bmod A)$. 
Proposition 2.7 Let $A \subseteq A P$ and $\sigma, \sigma^{\prime}$ be truth set sequences such that $\sigma \approx \sigma^{\prime}$ $(\bmod A)$ and $\phi$ a formula containing only atomic propositions in $A$. Then $\sigma \vDash \phi$ iff $\sigma^{\prime} \models \phi$.

Proof: Induction on the structure of $\phi$ [Lam83].

Corollary 2.8 Let $A \subseteq A P$ and $\phi$ be a formula containing only atomic propositions in $A$. Let $K$ and $K^{\prime}$ be (fair) Kripke-models such that for every (fair) fullpath $p$ in $K$ there is a (fair) fullpath $p^{\prime}$ in $K^{\prime \prime}$ such that $V(p) \stackrel{\approx}{\approx} V\left(p^{\prime}\right) \quad(\bmod A)$ and vice versa for all (fair) fullpaths $p^{\prime}$ in $K^{\prime}$. Then $K \vDash \phi$ if $K^{\prime} \vDash \phi$.

\section{Modules}

In this section we give the formal definitions of modules and the parallel composition operator. Each module $M$ has a set of its own variables that cannot be modified by the environment of $M$. In addition to its own variables a module may refer to a set of external variables and base decisions about its behaviour on their values. As in [MP81] a module is modelled by a directed graph, the states of which correspond to the execution states and the transitions to atomic actions. Here this model is simplified by allowing only boolean variables, by labelling the states of the model with the values of module's own variables, by dropping the assignment labels and by allowing only transition conditions consisting of a single proposition or its negation.

Definition 3.1 Let $A \subseteq A P$. By $\neg A$ we denote the set $\{\neg a \mid a \in A\}$, and by $L(A)$ the set $L(A)=A \cup \neg A \cup\{T\}$, where $T$ is an element not in $A$ or $\neg A$. If $a \in L(A)$ and $A^{\prime} \subseteq A$, we write $A^{\prime} \vDash a$ iff either $a=\top$ or $a \in A^{\prime}$ or $a=\neg a^{\prime}$ where $a^{\prime} \in A$ and $a^{\prime} \notin A^{\prime}$.

Definition 3.2 A module $M$ is an ordered 6-tuple $\left(A_{\circ}, A_{e}, S, I, R, V\right)$, where

- $A_{0} \subseteq A P$ is the set of atomic propositions owned by module $M$,

- $A_{e} \subseteq A P \backslash A_{o}$ is the set of external atomic propositions visible to $M$,

- $S$ is the set of states,

- $I \subseteq S$ is the set of initial states,

- $V: S \rightarrow P\left(A_{0}\right)$ is the truth valuation of module's own propositions, and

- $R \subseteq S \times L\left(A_{e}\right) \times S$ is the set of transitions. Each transition is labelled by a transition label which can be either trivially true $T$, an external atomic proposition or a negation of such. If $\left(s_{1}, l, s_{2}\right) \in R$, we write $s_{1} \stackrel{l}{\rightarrow} s_{2} \in R$.

Definition 3.3 A fair module $M_{f}$ is an ordered 7-tuple $M_{J}=\left(A_{o}, A_{\mathrm{e}}, S, I, R, V, F\right)$, where $\left(A_{o}, A_{e}, S, I, R, V\right)$ is a module and $F$ is a finite set of fairness sets $F=$ $\left\{f_{1}, \ldots f_{n}\right\}$ such that $\bigcup_{f_{i} \in F} f_{i}=R$. A basic fair module $M_{f}$ is a fair module such that $F=\{R\}$.

Modules may be combined using binary parallel composition \| which can be generalised to deal with $n$ modules in the standard fashion. Concurrency is represented by interleaving the atomic actions of the submodules. If a transition in one of the submodules is labelled by an atomic proposition owned by the other submodule, a corresponding transition in the combined module can be taken only if the proposition evaluates to true in the initial state of the transition. 
Definition 3.4 Let $M_{1}=\left(A_{o 1}, A_{e 1}, S_{1}, I_{1}, R_{1}, V_{1}\right)$ and $M_{2}=\left(A_{02}, \ldots, V_{2}\right)$ be modules such that $A_{o 1} \cap A_{o 2}=0$. The parallel composition of them, $M_{1} \| M_{2}$, is the module $\left(A_{o}, A_{e}, S, I, R, V\right)$ defined as follows:

- $A_{o}=A_{o 1} \cup A_{o 2}$

- $A_{e}=\left(A_{e 1} \cup A_{e 2}\right) \backslash A_{o}$

- $S=S_{1} \times S_{2}$

- $I=I_{1} \times I_{2}$

- $R=\left\{\left(s_{1}, t\right) \stackrel{\leftrightarrow}{\rightarrow}\left(s_{2}, t\right) \mid s_{1} \stackrel{\leftrightarrow}{\rightarrow} s_{2} \in R_{1}\right.$ and $\left.l \in L\left(A_{e}\right)\right\} U$

$\left\{\left(s_{1}, t\right) \stackrel{\sim}{\rightarrow}\left(s_{2}, t\right) \mid \exists l: s_{1} \stackrel{!}{\rightarrow} s_{2} \in R_{1}\right.$ and $l \in L\left(A_{o 2}\right)$ and $\left.V_{2}(t) \vDash l\right\} \cup$

$\left\{\left(s, t_{1}\right) \stackrel{\leftrightarrow}{\rightarrow}\left(s, t_{2}\right) \mid t_{1} \stackrel{\leftrightarrow}{\rightarrow} t_{2} \in R_{2}\right.$ and $\left.l \in L\left(A_{e}\right)\right\} \cup$

$\left\{\left(s, t_{1}\right) \stackrel{\top}{\rightarrow}\left(s, t_{2}\right) \mid \exists l: t_{1} \stackrel{\leftrightarrow}{\rightarrow} t_{2} \in R_{2}\right.$ and $l \in L\left(A_{o 1}\right)$ and $\left.V_{1}(s) \vDash l\right\}$

- $V\left(s_{1}, s_{2}\right)=V_{1}\left(s_{1}\right) \cup V_{2}\left(s_{2}\right)$

In the parallel composition of fair modules the fairness sets of the submodules are propagated to the resulting module. If a system is modelled by a parallel composition of basic fair modules, the definition guarantees that the fair fullpaths of the complete system are exactly those that are strongly fair with respect to each module.

Definition 3.5 Let $M_{f 1}=\left(A_{o 1}, A_{e 1}, S_{1}, I_{1}, R_{1}, V_{1},\left\{f_{11}, \ldots, f_{1 n_{1}}\right\}\right)$ and $M_{f 2}=$ $\left(A_{o 2}, \ldots, V_{2},\left\{f_{21}, \ldots, f_{2 n_{2}}\right\}\right)$ be fair modules. Then $M_{f 1} \| M_{f 2}$, is the fair module $\left(A_{0}, \ldots, V,\left\{f_{1}, \ldots, f_{n_{1}+n_{2}}\right\}\right)$ where $\left(A_{0}, \ldots, V\right)=\left(A_{01}, \ldots, V_{1}\right) \|\left(A_{02}, \ldots, V_{2}\right)$ and for $i=1, \ldots, n_{1}$

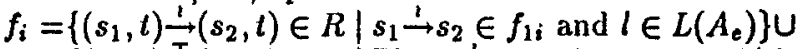

$\left\{\left(s_{1}, t\right) \stackrel{T}{\rightarrow}\left(s_{2}, t\right) \in R \mid \exists l: s_{1} \stackrel{!}{\rightarrow} s_{2} \in f_{1 i}\right.$ and $l \in L\left(A_{o 2}\right)$ and $\left.V_{2}(t) \vDash l\right\}$

and for $i=1, \ldots, n_{2}$

$f_{i+n_{1}}=\left\{\left(s, t_{1}\right) \underset{T}{\rightarrow}\left(s, t_{2}\right) \in R \mid t_{1} \stackrel{\prime}{\rightarrow} t_{2} \in f_{2 i}\right.$ and $\left.l \in L\left(A_{e}\right)\right\} \cup$

$\left\{\left(s, t_{1}\right) \stackrel{+}{\rightarrow}\left(s, t_{2}\right) \in R \mid \exists l: t_{1} \stackrel{\stackrel{\prime}{\rightarrow}}{\rightarrow} t_{2} \in f_{2 i}\right.$ and $l \in L\left(A_{o 1}\right)$ and $\left.V_{1}(s) \vDash l\right\}$

The availability of a transition in a module that does not have any nontrivial transition conditions cannot be influenced by its environment. Therefore, we treat such a module as corresponding to a complete system, and interpret formulas in the naturally induced Kripke-model.

Definition 3.6 Let $M=\left(A_{0}, A_{e}, S, I, R, V\right)$ be a module. $M$ is closed iff $A_{e}=0$ and the Kripke-model $K(M)$ corresponding to the closed module $M$ is defined as $K(M)=\left(S, I, R_{K}, V\right)$, where $\left(s_{1}, s_{2}\right) \in R_{K}$ iff $s_{1} \stackrel{⿱ 乛}{\rightarrow} s_{2} \in R$.

Let $M_{f}=\left(A_{o}, \ldots, V,\left\{f_{1}, \ldots, f_{n}\right\}\right)$ be a fair module. $M_{f}$ is closed iff $A_{c}=0$ and the fair Kripke-model $K\left(M_{f}\right)$ corresponding to $M_{f}$ is defined as $K\left(M_{f}\right)=$ $\left(S, I, R_{K}, V,\left\{f_{K 1}, \ldots, f_{K n}\right\}\right)$, where $R_{K}$ is as above and for all $1 \leq i \leq n:\left(s_{1}, s_{2}\right) \in$ $f_{K i}$ iff $s_{1} \stackrel{\leftarrow}{\rightarrow} s_{2} \in f_{i}$.

A formula $\phi$ is true of a closed (fair) module $M, M \vDash \phi$, iff $K(M) \vDash \phi$.

\section{Compositional model checking}

The basic method of compositional verification applied in this paper is that of [MP91]: when verifying that $\phi$ holds of $M_{1} \| M_{2}$, find $\phi_{1}$ and $\phi_{2}$ such that $\phi_{i}$ holds of $M_{i}$ in any environment and $\models \phi_{1} \wedge \phi_{2} \Rightarrow \phi$.

Definition 4.1 A formula $\phi$ is modularly true of $M$ iff $M \| M^{\prime} \vDash \phi$ for all modules $M^{\prime}$ such that $M \| M^{\prime}$ is closed. If this is the case we write $M=\stackrel{m}{=} \phi$. 
The correctness of the verification method is asserted by the following result, which can be generalised to $n$ processes.

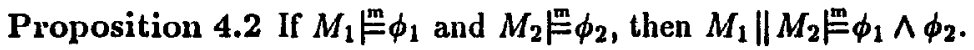

The aim now is to find a method for verifying that $M=\phi$. This can be done by constructing a Kripke-model $K$ on the basis of $M$ so that $M=\phi$ iff $K \vDash \phi$ and by applying the standard linear temporal logic model checking algorithm [LP85] to check whether $K \vDash \phi$ holds. A straighforward approach is to construct a single environment of $M$ so that it exhibits all the possible behaviours that any environment of $M$ can produce and to combine this environment with $M$.

Definition 4.3 Let $M=\left(A_{o}, \ldots, V\right)$ be a module. The chaotic environment of $M$, $\operatorname{ce}(M)$ is the module $\left(A_{e}, \emptyset, P\left(A_{e}\right), P\left(A_{\epsilon}\right), P\left(A_{e}\right) \times P\left(A_{e}\right), V^{\prime}\right)$, where $V^{\prime}(s)=s$.

Proposition 4.4 Let $M=\left(A_{o}, \ldots, V\right)$ be a module and $\phi$ a formula such that every atomic proposition occurring in $\phi$ is in $A_{o} \cup A_{e}$. Then $M{ }^{m}=\phi$ iff $M \| c e(M) \vDash \phi$.

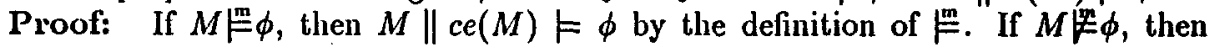
there is an $M^{\prime}$ and a path $p_{1}$ in $K_{1}=K\left(M \| M^{\prime}\right)$ such that $V_{1}\left(p_{1}\right) \not \phi$ holds. If $p_{1}=\left(\left(s_{1}, t_{1}\right),\left(s_{2}, t_{2}\right), \ldots\right)$, then $p_{2}=\left(\left(s_{1}, V_{1}\left(t_{1}\right) \cap A_{e}\right),\left(s_{2}, V_{1}\left(t_{2}\right) \cap A_{e}\right), \ldots\right)$ is a path in $K_{2}=K(M \| c e(M))$. What is more, $V_{1}\left(p_{1}\right) \approx V_{2}\left(p_{2}\right)\left(\bmod A_{o} \cup A_{\theta}\right)$. By 2.7, $V_{2}\left(p_{2}\right) \nvdash \phi$ which implies $M \| c e(M) \nvdash \phi$.

Please note that the set $A_{e}$ can always be extended so that every atomic proposition occurring in $\phi$ is in $A_{o} \cup A_{e}$. Therefore, without loss of generality we suppose that this is the case in the following.

Checking whether $M=\mid m$ can be done by checking that $M \| c e(M) \vDash \phi$. This is exactly the method proposed in [MP91], where a transition system equivalent to $K(M \| c e(M))$ is denoted by $S_{M}$. As $|M \| \operatorname{ce}(M)|=|M| \cdot 2^{\left|A_{e}\right|}$, the model checking will take $O\left(|M| \cdot 2^{\left|A_{e}\right|} \cdot 2^{c \cdot|\phi|}\right)$ time. This is sensible only if $\left|A_{e}\right|<\log \left(\left|M^{\prime}\right|\right)$, where $M^{\prime}$ is the actual environment in which $M$ is to work.

In this naive approach we are, in fact, doing a lot of unnecessary work. What we are actually interested in is the validity of $\phi$ in all the fullpaths of all the systems consisting of $M$ and an environment $M^{\prime}$. The only influence of the external atomic propositions not occurring in $\phi$ is the availability of some transitions in some states and, consequently, the existence or nonexistence of some fullpaths. However, if a certain transition is labelled by an external atomic proposition, there is always both an environment in which the transition is disabled and an environment in which the transition is enabled. The first possibility is always present, anyway, since an infinite execution of the environment unfair to $M$ might prevent $M$ from taking a transition even if it were enabled. Therefore, we do not need to pay any attention to whether transition conditions not occurring in $\phi$ are true or false, and we may simply drop them.

Definition 4.5 Let $M=\left(A_{0}, \ldots, V\right)$ be a module and $A \subseteq A_{o} \cup A_{e}$. The satisfiability graph corresponding to $M$ and $A, s g(M, A)$, is the Kripke-model $\left(S_{g}, I_{g}, R_{g}, V_{g}\right)$ where

$$
\text { - } \begin{aligned}
S_{g}= & S \times P\left(A \backslash A_{o}\right) \\
\text { - } I_{g}=I \times P\left(A \backslash A_{o}\right) & I \times R_{g}= \\
\text { - } R_{g}=\left\{\left(\left(s, A_{1}\right),\left(s, A_{2}\right)\right) \in S_{g} \times S_{g}\right\} \cup & \left\{\left(\left(s_{1}, A_{1}\right),\left(s_{2}, A_{1}\right)\right) \in S_{g} \times S_{g} \mid \exists l: s_{1} \stackrel{\leftrightarrow}{\rightarrow} s_{2} \in R \text { and } l \notin L(A) \text { or } A_{1} \vDash l\right\}
\end{aligned}
$$


- $V_{y}\left(s, A_{1}\right)=V(s) \cup A_{1}$

Proposition 4.6 Let $M=\left(A_{o}, \ldots, V\right)$ be a module, $A \subseteq A_{o} \cup A_{e}$ and $\phi$ a formula containing only atomic propositions from $A$. Then $M=\bar{\phi}$ iff $s g(M, A) \vDash \phi$.

Proof: By 4.4, $M=\phi$ iff $M \| c e(M) \vDash \phi$. Here it is shown that $M \| c e(M) \vDash \phi$ iff $\boldsymbol{s g}(M, A) \vDash \phi$.

Let us denote $M \| c e(M)$ by $M_{c}=\left(A_{c a}, A_{c e}, S_{c}, I_{c}, R_{c}, V_{c}\right)$ and $s g(M, A)$ by $K_{g}=\left(S_{g}, I_{g}, R_{g}, V_{g}\right)$. The result is obtained by showing that given any fullpath $p$ in $K\left(M_{c}\right)$ there is a fullpath $p^{\prime}$ in $K_{g}$ such that $V_{c}(p) \approx V_{g}\left(p^{\prime}\right)(\bmod A)$ and vice versa, and by applying 2.8 .

Assume that $K\left(M_{c}\right)$ has a fullpath $p$. From the definitions of $K()$ and $M_{c}$ it is known that $p$ is of the form $p=\left(\left(s_{1}, A_{1}\right),\left(s_{1}, A_{2}\right), \ldots\right)$, where each $s_{i} \in S, A_{i} \in$ $P\left(A_{e}\right)$, and either $s_{i}=s_{i+1}$ or $A_{i}=A_{i+1}$ and there is an $l$ such that $s_{i} \stackrel{\dot{ }}{\rightarrow} s_{i+1} \in R$ and $A_{i} \vDash l$ l. If $s_{i}=s_{i+1}$, it is clear that $\left(\left(s_{i}, A_{i} \cap A\right),\left(s_{i+1}, A_{i+1} \cap A\right)\right) \in R_{g}$. If $s_{i} \neq s_{i+1}$, then $A_{i}=A_{i+1}$ and either $l \notin L(A)$ or $l \in L(A)$ and $A_{i} \cap A=l$. In both cases $\left(\left(s_{i}, A_{i} \cap A\right),\left(s_{i+1}, A_{i+1} \cap A\right)\right) \in R_{g}$ follows. As $\left(s_{1}, A_{1} \cap A\right) \in I_{g}$, $p^{\prime}=\left(\left(s_{1}, A_{1} \cap A\right),\left(s_{2}, A_{2} \cap A\right), \ldots\right)$ is a fullpath in $K_{g}$. What is more, $V_{\mathrm{c}}(p) \approx V_{g}\left(p^{\prime}\right)$ $(\bmod A)$ since $V_{c}\left(\left(s_{i}, A_{i}\right)\right) \cap A=\left(V\left(s_{i}\right) \cup A_{i}\right) \cap A=V_{g}\left(\left(s_{i}, A_{i} \cap A\right)\right) \cap A$.

Assume now that $K_{g}$ has a fullpath $p$. From the definition of $K_{g}$ it is known that $p=\left(\left(s_{1}, A_{1}\right),\left(s_{2}, A_{2}\right), \ldots\right)$ where for each $\left(\left(s_{i}, A_{i}\right),\left(s_{i+1}, A_{i+1}\right)\right)$ either $s_{i}=s_{i+1}$ or $A_{i}=A_{i+1}$ and there is an $l$ such that $s_{i} \rightarrow s_{i+1} \in R$ and either $A_{i} \vDash l$ or $l \notin L(A)$. If $s_{i}=s_{i+1}$, it is clear that $\left(s_{i}, A_{i}\right) \stackrel{\mathcal{T}}{\rightarrow}\left(s_{i+1}, A_{i+1}\right) \in R_{c}$. If $A_{i}=A_{i+1}$ and $A_{i}=l$, $\left(s_{i}, A_{i}\right) \stackrel{\sim}{\rightarrow}\left(s_{i+1}, A_{i+1}\right) \in R_{c}$, again. If $A_{i}=A_{i+1}$ and $l \notin L(A)$ and $l$ is of the form $\neg l_{1}, l_{1} \notin A_{i}$ which implies that $A_{i} \vDash l$. Consequently, $\left(s_{i}, A_{i}\right)^{\mathrm{T}} \rightarrow\left(s_{i+1}, A_{i+1}\right) \in$ $R_{c}$. Finally, if $A_{i}=A_{i+1}$ and $l \notin L(A)$ and $l$ is not of the form $\neg l_{1}, R_{c}$ has the transitions $\left(s_{i}, A_{i}\right)^{\stackrel{I}{\rightarrow}}\left(s_{i}, A_{i} \cup\{l\}\right) \stackrel{\sim}{\rightarrow}\left(s_{i+1}, A_{i+1} \cup\{l\}\right) \stackrel{\sim}{\rightarrow}\left(s_{i+1}, A_{i+1}\right)$. Therefore, for each transition of $p$ in $K_{g}$ we can construct a sequence of transitions in $R_{c}$ so that they contain the same initial and final states. The required path $p^{\prime}$ in $K\left(M_{c}\right)$ is constructed by joining these sequences together. By the structure of $p^{\prime}, V_{g}(p) \approx V_{c}\left(p^{\prime}\right)$ $(\bmod A)$ as well.

By proposition 4.6 we can check whether $M \stackrel{m}{=} \phi$ by taking $A$ as the set containing only the atomic propositious in $\phi$ and by checking whether $s g(M, A) \vDash \phi$. Noticing that $|s g(M, A)|=|M| \cdot 2^{\left|A \backslash A_{o}\right|}$ and that $\left|A \backslash A_{o}\right|$ is limited by $|\phi|$, we acquire an upper limit $|M| \cdot 2^{|\phi|}$ to the size of the satisfiability graph. Since the satisfiability graph can be constructed in a time linear to its size, the model checking takes $O\left(|M| \cdot 2^{|\phi|} \cdot 2^{c \cdot|\phi|}\right)$, i.e. $O\left(|M| \cdot 2^{c \cdot|\phi|}\right)$ time. The total time requirement of the method consists of three parts: verifying that $M_{1} \stackrel{m}{=} \phi_{1}$, which takes $O\left(\left|M_{1}\right| \cdot 2^{c \cdot\left|\phi_{1}\right|}\right)$ time, verifying that $\left.M_{2}\right|^{2} \phi_{2}$, which takes $O\left(\left|M_{2}\right| \cdot 2^{c \mid} \cdot \phi_{2} \mid\right)$ time, and verifying that $\vDash \phi_{1} \wedge \phi_{2} \Rightarrow \phi$, which takes $O\left(2^{c \cdot\left(\left|\phi_{1}\right|+\left|\phi_{2}\right|+|\phi|\right)}\right)$ time [LPZ85]. Assuming $\left|\phi_{1}\right| \approx\left|\phi_{2}\right|$, the total time needed is thus $O\left(\left(\left|M_{1}\right|+\left|M_{2}\right|+2^{c \cdot|\phi|}\right) \cdot 2^{c \cdot\left|\phi_{1}\right|}\right)$. Furthermore, if $|\phi| \approx\left|\phi_{1}\right|$, this is $O\left(\left(\left|M_{1}\right|+\left|M_{2}\right|\right) \cdot 2^{c \cdot|\phi|}\right)$.

This should be contrasted with the time requirement $O\left(\left|M_{1}\right| \cdot\left|M_{2}\right| \cdot 2^{c \cdot|\phi|}\right)$ of the simple approach of just constructing the whole state space of the system and then running the model checker on it. If $M_{1}$ and $M_{2}$ are large and the required lemmas $\phi_{1}$ and $\phi_{2}$ relatively short, the compositional method presented here can therefore yield substantial savings. As an additional note, the restriction that transitions are labelled by single propositions or their negations can be dropped in favour of arbitrary formulas in the disjunctive normal form without affecting the time requirement. 


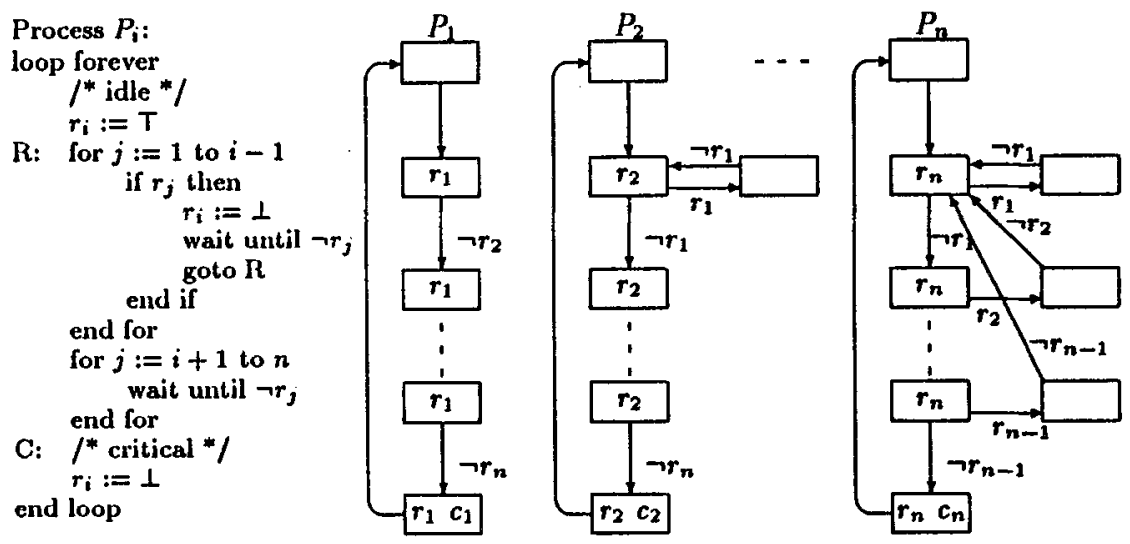

Figure 1: Mutual exclusion protocol

Although no systematic way of finding suitable lemmas is developed in this paper, the issue is naturally very important for real-world applications. Ideally, when creating a concurrent system the designer sets some requirements to each module so that the requirements to the complete system are met if the requirements to the individual modules are met. These requirements naturally form a good basis for the required lemmas. The applications of the method are best illustrated by an example.

Example 4.7 Figure 1 contains a textual description of a mutual exclusion protocol and the corresponding module graphs. Each state in the picture is labelled by the atomic propositions true in that state. The only propositions shown are $\boldsymbol{r}_{i}$, which has the meaning process $i$ requests access to the critical section, and $c_{i}$ meaning process $i$ is in the critical section. For each module $P_{i}, A_{o i}=\left\{r_{i}, c_{i}\right\}$ and $A_{e i}=$ $\left\{r_{j} \mid 1 \leq j \leq n, j \neq i\right\}$. An obvious requirement to the system is that two processes are never together in the critical section, i.e. that $\bigwedge_{i \neq j} \square\left(\neg c_{j} \vee \neg c_{i}\right)$.

If we analyse a system consisting of $n$ such processes, the number of global states is $O\left(n^{n}\right)$. This means that e.g. for $n=32$ the number of global states would be in the range $2^{160}$, which rules out straightforward model checking.

Let us denote by $\phi$ the formula $\square\left(\neg c_{j} \vee \neg c_{i}\right)$ for some fixed $i$ and $j$. If we can show that $\phi$ holds for all $i \neq j$, the required result follows. As lemma $\phi_{1}$ we may take the formula $\neg r_{i} \wedge \square\left(c_{i} \Rightarrow r_{i}\right) \wedge \square\left(\neg r_{i} \Rightarrow\left(\neg c_{i}\right) \mathcal{U}_{w}\left(r_{i} \wedge \neg r_{j}\right)\right)$ and as lemma $\phi_{2}$ the same formula with $i$ :s and $j$ :s reversed. It is easy to check that $\vDash \phi_{1} \wedge \phi_{2} \Rightarrow \phi$.

If we tried to verify that $P_{i}=^{\mathrm{m}} \phi_{1}$ using the naive method of 4.4 , the size of the resulting Kripke-model would have a minimum of $2^{36}$ states. Even this would very likely be infeasible.

When verifying that $P_{i} \models^{\text {un }} \phi_{1}$ using the satisfiability graph construction, the only external proposition that we need to keep track of is $r_{j}$, and consequently the satisfiability graph has a maximum of 128 states. The same applies to verifying that $P_{j}{ }^{\prime m} \phi_{2}$. The advantages of the method are clear in this case. 


\section{Fair concurrency}

Despite the advantages of the verification method presented in the previous section, it still suffers from an important drawback: when two modules are combined in parallel, the resulting system in not required to be fair with respect to both modules. One counter-intuitive result of this is that even if $M$ is a closed module, $M \vDash \phi$ does not necessarily imply $M \stackrel{m}{=} \phi$. In this section we present a remedy to these problems in the form of a variant of the satisfiability graph construction which can takes fairness requirements into consideration.

Definition 5.1 A formula $\phi$ is modularly true of a fair module $M_{f}$, i.e. $M_{f} \stackrel{\text { im }}{=} \phi$, iff $M_{f} \| M_{f}^{\prime} \vDash \phi$ for all fair modules $M_{f}^{\prime}$ such that $M_{f} \| M_{f}^{\prime}$ is closed.

The following result states that for a closed fair module it does not matter whether $M$ is considered in or without an environment, i.e. that the fairness requirements remove the problem stated above.

Proposition 5.2 If $M_{f}$ is a closed fair module, then $M_{f} \stackrel{f m}{=} \phi$ iff $M_{f} \vDash \phi$.

Definition 5.3 Let $M_{f}=\left(A_{o}, \ldots, F\right)$ be a fair module. The fair chaotic environment of $M_{f}, \operatorname{ce}\left(M_{f}\right)$ is the fair module $\left(A_{o}^{\prime}, \ldots, V^{\prime},\left\{P\left(A_{e}\right) \times P\left(A_{e}\right)\right\}\right)$, where $A_{o}^{\prime}, \ldots, V^{\prime}$ are as in 4.3 .

Proposition 5.4 Let $M_{f}=\left(A_{o}, \ldots, F\right)$ be a fair module and $\phi$ a formula such that every atomic proposition occurring in $\phi$ is in $A_{o} \cup A_{e}$. Then $M_{f}$ 作 $\phi$ iff $M_{f} \|$ $c e\left(M_{J}\right) \vDash \phi$.

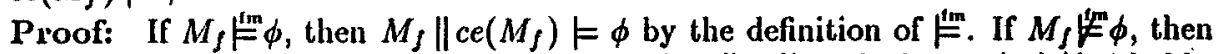
there is an $M_{j}^{\prime}$ and a fair path $p_{1}$ in $K_{1}=K\left(M_{f} \| M_{\jmath}^{\prime}\right)$ such that $V_{1}\left(p_{1}\right) \notin \phi_{f}$ holds. As in 4.4 there is a path $p_{2}$ in $K_{2}=K\left(M_{f} \| c e\left(M_{f}\right)\right)$ such that $V_{1}\left(p_{1}\right) \approx V_{2}\left(p_{2}\right)$ $\left(\bmod A_{0} \cup A_{e}\right)$. As $p_{1}$ is fair with respect to every faimess set $f \in F, p_{2}$ is, by its structure, also fair with respect to all the corresponding fairness sets of $K_{2}$. However, $p_{2}$ is not necessarily fair with respect to the one fairness set of $K_{2}$ corresponding to the single fairness set of $c e\left(M_{J}\right)$. This can be rectified by interleaving an infinite number of non-state-changing transitions of $c e\left(M_{f}\right)$ into $p_{2}$ without affecting $V_{1}\left(p_{1}\right) \stackrel{\nexists n}{\approx} V_{2}\left(p_{2}\right) \quad\left(\bmod A_{0} \cup A_{e}\right)$.

In the construction of the satisfiability graph we have now the additional concern of discerning fair paths from unfair ones. What essentially happens when we move from the naive approach with the chaotic environment to the satisfiability graph is grouping together states $\left(s, A^{\prime}\right)$ where the $A^{\prime}$ :s differ only with respect to propositions not in $A$. This grouping, however, may introduce new dependencies between the enabled fairness sets, and may therefore have unwanted effects on which paths are regarded fair. The way to do away with these dependencies in order to reflect the original structure faithfully is to make as many copies of a state $\left(s, A^{\prime}\right)$ as there are possible combinations of enabled fairness sets in the module, i.e. 2 copies in the case of a basic fair module, and $2^{|F|}$ copies in the general case. Each copy $\left(s, A^{\prime}, F^{\prime}\right)$, where $F^{\prime} \subseteq F$, represents the possibility that in the chaotic environment it is possible to reach a state $\left(s, A^{\prime \prime}\right)$ such that $A^{\prime \prime} \cap A=A^{\prime}$ and all the enabled fairness sets are in $F^{\prime}$. 
Definition 5.5 Let $M_{J}=\left(A_{0}, \ldots, F\right)$ be a fair module, $A \subseteq A_{0} \cup A_{e}, A_{1} \subseteq A_{e} \cap A$, $A_{1}^{\prime} \subseteq A_{e}, s \in S$ and $F_{1} \subseteq F$. We say that $\left(s, A_{1}^{\prime}\right)$ is an $F_{1}$-enabled $A$-extension of $\left(s, A_{1}\right)$ iff $A_{1}^{\prime} \cap A=A_{1}$ and for every $f \in F$ the following holds: if there are $l$ and $s^{\prime}$ such that $s \rightarrow s^{\prime} \in f$ and $A_{1}^{\prime} \vDash l$, then $f \in F_{1}$.

Definition 5.6 Let $M_{f}=\left(A_{0}, \ldots, F\right)$ be a fair module, where $F=\left\{f_{1}, \ldots, f_{n}\right\}$, and $A \subseteq A_{o} \cup A_{e}$. The fair satisfiability graph corresponding to $M_{f}$ and $A, s g\left(M_{f}, A\right)$ is the fair Kripke-model $\left(S_{g}, I_{g}, R_{g}, V_{g}, F_{g}\right)$ defined as follows:

- $S_{g}=\left\{\left(s, A_{1}, F_{1}\right) \in S \times P\left(A \backslash A_{o}\right) \times P(F)\right\}$

$\exists A_{1}^{\prime}:\left(s, A_{1}^{\prime}\right)$ is an $F_{1}$-enabled $A$-extension of $\left.\left(s, A_{1}\right)\right\}$

- $I_{g}=\left(I \times P\left(A \backslash A_{o}\right) \times P(F)\right) \cap S_{g}$

- $R_{g}=\bigcup_{i=1}^{n+1} f_{g i}$, where for all $1 \leq i \leq n$

$f_{g i}=\left\{\left(\left(s_{1}, A_{1}, F_{1}\right),\left(s_{2}, A_{2}, F_{2}\right)\right) \in S_{g} \times S_{g} \mid f_{i} \in F_{1}\right.$ and $A_{1}=A_{2}$ and

$\exists A_{1}^{\prime}: \exists l: A_{1}^{\prime} \vDash l$ and $s_{1} \rightarrow s_{2} \in f_{i}$ and

$\left(s_{1}, A_{1}^{\prime}\right)$ is an $F_{1}$-enabled $A$-extension of $\left(s_{1}, A_{1}\right)$ and

$\left(s_{2}, A_{1}^{\prime}\right)$ is an $F_{2}$-enabled $A$-extension of $\left.\left(s_{2}, A_{2}\right)\right\}$

$f_{g n+1}=\left\{\left(\left(s, A_{1}, F_{1}\right),\left(s, A_{2}, F_{2}\right)\right) \in S_{g} \times S_{g}\right\}$

- $V_{g}\left(s, A_{1}, F_{1}\right)=V(s) \cup A_{1}$

- $F_{g}=\left\{f_{g 1}, \ldots, f_{g n}, f_{g n+1}\right\}$

Proposition 5.7 Let $M_{J}=\left(A_{0}, \ldots, F\right)$ be a fair module, where $F=\left\{f_{1}, \ldots, f_{n}\right\}$, and $A \subseteq A_{o} \cup A_{\mathrm{e}}$. If $\phi$ is a formula containing only atomic propositions from $A$, then $M_{f} \vDash \phi$ iff $s g\left(M_{f}, A\right) \vDash \phi$.

Proof: In the proof we use following notation: $M_{f} \| c e\left(M_{f}\right)$ is denoted by $M_{c}=$ $\left(A_{c o}, A_{c e}, S_{c}, I_{c}, R_{c}, V_{c}, F_{c}\right)$, where $F_{c}=\left\{f_{c 1}, \ldots, f_{c n+1}\right\}$, and $s g\left(M_{f}, A\right)$ by $K_{g}=$ $\left(S_{g}, I_{g}, R_{g}, V_{g}, F_{g}\right)$, where $F_{g}=\left\{f_{g 1}, \ldots, f_{g n+1}\right\}$. The indexing of the fairness sets $F_{c}$ and $F_{g}$ is supposed to correspond to the indexing of the original fairness set $F$. If $\left(s_{i}, A_{i}\right) \in S_{c}$, then $e n\left(s_{i}, A_{i}\right)=\left\{f_{j} \in F \mid f_{c j}\right.$ is enabled in $\left.\left(s_{i}, A_{i}\right)\right\}$.

The following facts are direct consequences of the definitions:

1 If $\left(\left(s_{i}, A_{i}\right),\left(s_{i+1}, A_{i+1}\right)\right) \in f_{c j}$, then $\left(\left(s_{i}, A_{i} \cap A, e n\left(s_{i}, A_{i}\right)\right),\left(s_{i+1}, A_{i+1} \cap A, e n\left(s_{i+1}, A_{i+1}\right)\right)\right) \in f_{g j}$.

2 If $1 \leq j \leq n$, then $f_{g j}$ is enabled in $\left(s_{i}, A_{i}, F_{i}\right) \in S_{g}$ only if $f_{j} \in F_{i}$. Consequently, if $f_{g j}$ is enabled in $\left(s_{i}, A_{i} \cap A, e n\left(s_{i}, A_{i}\right)\right)$ then $f_{c j}$ is enabled in $\left(s_{i}, A_{i}\right)$.

3 If $\left(\left(s_{i}, A_{i}, F_{i}\right),\left(s_{i+1}, A_{i+1}, F_{i+1}\right)\right) \in f_{g j}$, then there exist $A_{i}^{\prime \prime}$ and $A_{i+1}^{\prime}$ such that $\left(\left(s_{i}, A_{i}^{\prime \prime}\right),\left(s_{i+1}, A_{i+1}^{\prime}\right)\right) \in f_{c j}, A_{i}^{\prime \prime} \cap A=A_{i}, A_{i+1}^{\prime} \cap A=A_{i+1}$, and for all $1 \leq k \leq n+1:$ if $f_{k} \in e n\left(s_{i}, A_{i}^{\prime \prime}\right)$ then $f_{c k}$ is enabled in $\left(s_{i}, A_{i}, F_{i}\right)$, and if $f_{k} \in \operatorname{en}\left(s_{i+1}, A_{i+1}^{\prime}\right)$ then $f_{c k}$ is enabled in $\left(s_{i+1}, A_{i+1}, F_{i+1}\right)$.

As in 4.6, the proof proceeds by showing that if $K\left(M_{c}\right)$ has a fair fullpath $p$ then $K_{g}$ has a fair fullpath $p^{\prime}$ such that $V_{c}(p) \approx V_{g}\left(p^{\prime}\right) \quad(\bmod A)$ and vice versa.

Assume that $p=\left(\left(s_{1}, A_{1}\right),\left(s_{2}, A_{2}\right), \ldots\right)$ is a fair fullpath of $K\left(M_{c}\right)$. The required $p^{\prime}$ can be obtained as $p^{\prime}=\left(\left(s_{1}, A_{1} \cap A, e n\left(s_{1}, A_{1}\right)\right),\left(s_{2}, A_{2} \cap A\right.\right.$,en $\left.\left.\left(s_{2}, A_{2}\right)\right), \ldots\right)$. By 1 above and by the definition of $R_{g}, p^{\prime}$ is a fullpath. As $p$ is fair, 1 and 2 imply that $p^{\prime}$ is fair as well. $V_{c}(p) \stackrel{\nexists d}{\approx} V_{g}\left(p^{\prime}\right)(\bmod A)$ is established as in 4.6.

Assume that $p=\left(\left(s_{1}, A_{1}, F_{1}\right),\left(s_{2}, A_{2}, F_{2}\right), \ldots\right)$ is a fair fullpath of $K_{g}$. The required $p^{\prime}$ can be obtained as $p^{\prime}=\left(\left(s_{1}, A_{1}^{\prime \prime}\right),\left(s_{2}, A_{2}^{\prime}\right),\left(s_{2}, A_{2}^{\prime \prime}\right),\left(s_{3}, A_{3}^{\prime}\right), \ldots\right)$, where $A_{i}^{\prime}$ and $A_{i}^{\prime \prime}$ are as in 3 above. Note that for every transition in $p$ there are two transitions in $p^{\prime}$. By 3 above, $p^{\prime}$ is a fullpath. As $p$ is fair, 3 implies that $p^{\prime}$ is fair as well. Finally, the definitions of $A_{i}^{\prime}$ and $A_{i}^{\prime \prime}$ used in constructing $p^{\prime}$ guarantee that $V_{g}(p) \ddot{\approx} V_{c}\left(p^{\prime}\right)(\bmod A)$. 
The size of the satisfiability graph is $|M| \cdot 2^{|F|} \cdot 2^{|A| A_{0} \mid}$. In constructing the satisfiability graph the essential step is deciding whether $s_{1} \stackrel{\leftrightarrow}{\rightarrow} s_{2} \in f_{i}$ implies $\left(\left(s_{1}, A_{1}, F_{1}\right),\left(S_{2}, A_{2}, F_{2}\right)\right) \in f_{g i}$. This can be done by checking whether formula

$$
l \wedge \bigwedge_{J \in F \backslash F_{1}} \bigwedge_{\left(s_{1}, a, s_{1}^{\prime}\right) \in J} \neg a \wedge \bigwedge_{J \in F \backslash F_{2}} \bigwedge_{\left(s_{2}, a, s_{j}^{\prime}\right) \in f} \neg a
$$

is satisfiable, which can be done in linear time. Therefore, the satisfiability graph can be constructed in a time linear to its size. As checking the truth of $\phi$ over $s g\left(M_{\rho}, A\right)$ takes $O\left(|F| \cdot\left|s g\left(M_{f}, A\right)\right| \cdot 2^{c \cdot|\phi|}\right)$ time, checking modular truth of $\phi$ in a fair module $M_{f}$ requires $O\left(|F| \cdot\left|M_{J}\right| \cdot 2^{|F|} \cdot 2^{|\phi|} \cdot 2^{c \cdot|\phi|}\right)$, i.e. $O\left(\left|M_{f}\right| \cdot 2^{c \cdot|F|} \cdot 2^{c \cdot|\phi|}\right)$ time. This is less than that of the naive approach as long as $|F|<\left|A P_{e}\right| A \mid$. Although the time requirement is exponential in $|F|$, this is unlikely to cause problems in practice as $|F|$ is typically very low.

Fairness between concurrent processes in a system is a special case of the more general fairness notion treated above. In order to model it only basic fair modules are needed. These have the property that all the transitions of a module form a single fairness set, i.e. that $|F|=1$. In this case the time requirement reduces to $O\left(\left|M_{f}\right| \cdot 2^{c \cdot|\phi|}\right)$, which means that we can answer the question about the truth of $\phi$ over module $M$ in all strongly fair environments in the same time as over $M$ in all environments.

As the last issue we discuss briefly the existence of the lemmas $\phi_{1}$ and $\phi_{2}$, and draw atlention to a particular case in which we can be sure of their existence. Intuitively the following result shows that if every state of a module can be uniquely characterised by the atomic propositions true in that state, the lemmas always exist. This should come as no suprise, as the characterisability means that the structure of the modules can be axiomatised.

Proposition 5.8 Let $M_{1}, M_{2}$ be (fair) modules such that both $V_{1}$ and $V_{2}$ are injective, i.e. that for all $s, s^{\prime} \in S_{1}: V_{1}(s)=V_{1}\left(s^{\prime}\right)$ implies $s=s^{\prime}$ and alike for $V_{2}$.

If $M_{1} \| M_{2} \stackrel{i m}{=} \phi$, there exist formulas $\phi_{1}$ and $\phi_{2}$ such that $M_{1} \stackrel{f m}{=} \phi_{1}, M_{2} \stackrel{f m}{=} \phi_{2}$ and $\models \phi_{1} \wedge \phi_{2} \Rightarrow \phi$.

Proof: Thanks to the characterisability of $M_{i}$ we can construct formulas $\operatorname{ch}\left(M_{i}\right)$ so that they completely describe the behaviour of $M_{i}$.

$$
\begin{aligned}
& \operatorname{ch}(s)=\bigwedge_{a \in V(s)} a \wedge \bigwedge_{a \in A_{0} \backslash V(s)} \neg a \\
& \operatorname{ch}(R)=\square \bigwedge_{s \in S}(\operatorname{ch}(s) \Rightarrow \operatorname{tr}(s)) \\
& \operatorname{tr}(s)=\operatorname{ch}(s) U_{w}\left(\bigvee_{\left(s, 1, s^{\prime}\right) \in R}\left(\operatorname{ch}\left(s^{\prime}\right) \wedge l\right)\right) \\
& \operatorname{ch}\left(f_{i}\right)=(\square \diamond \bigvee(\operatorname{ch}(s) \wedge l)) \Rightarrow\left(\square \diamond \bigvee\left(\operatorname{ch}(s) U \operatorname{ch}\left(s^{\prime}\right)\right)\right) \\
& \left(s, l, s^{\prime}\right) \in f_{i} \quad\left(s, l, s^{\prime}\right) \in f_{i} \\
& \operatorname{ch}(M)=\square \bigvee_{s \in S} \operatorname{ch}(s) \wedge \bigvee_{s \in I} \operatorname{ch}(s) \wedge \operatorname{ch}(R) \wedge \operatorname{ch}(F) \\
& \operatorname{int}\left(M, M^{\prime}\right)=\bigwedge_{a \in L\left(A_{0}\right) a^{\prime} \in L\left(A_{o}^{\prime}\right)}^{s \in S} a \wedge a^{\prime} \Rightarrow\left(\left(a \wedge a^{\prime}\right) U_{w}\left(\left(\neg a \wedge a^{\prime}\right) \vee\left(a \wedge \neg a^{\prime}\right)\right)\right) \\
& \operatorname{ch}(F)=\bigwedge_{f_{i} \in F} \operatorname{ch}\left(f_{i}\right)
\end{aligned}
$$

(The formula $\operatorname{int}\left(M, M^{\prime}\right)$ decrees that two propositions owned by different modules cannot change their truth-value at the same time.) Now $M_{1} \stackrel{\operatorname{sm}}{=} \operatorname{ch}\left(M_{1}\right) \wedge \operatorname{int}\left(M_{1}, M_{2}\right)$ and alike for $M_{2}$. It remains to show that if $M_{1}\left\|M_{2}\right\|^{i m} \phi$ then $\vDash \operatorname{ch}\left(M_{1}\right) \wedge$ $\operatorname{inl}\left(M_{1}, M_{2}\right) \wedge \operatorname{ch}\left(M_{2}\right) \Rightarrow \phi$. This can be done by supposing that, on the contrary, there were a Kiripke-model $K$ and a path $p$ falsifying the implication, and by 
constructing a path $p^{\prime}$ in $\left(M_{1} \| M_{2}\right) \| c e\left(M_{1} \| M_{2}\right)$ falsifying $\phi$ as well, and thus creating a contradiction.

It is clear that the lemmas constructed in the proof are of purely theoretical value due to their length. Ilowever, knowing that the method is complete in the sense that any property can be verified by it naturally adds confidence to it.

\section{Discussion}

In this paper we have presented algorithms deciding the truth of a nexttime-less linear temporal logic formula $\phi$ over a distributed-variable module $M$ in all environments and in all fair environments. An interesting result obtained is that the time-complexity of checking whether a property holds of a module in all environments is essentially no higher than that of checking whether it holds without any environment. The method of modular verification suggested here seems to be very promising. However, what is still needed for a fully-fledged system is a methodology for creating the required lemmas.

\section{References}

[Bar86] Barringer, H.: Using Temporal Logic in the Compositional Specification of Concurrent Systems, in Galton, A. (ed.): Temporal Logics and Their Applications, Academic Press, 1987, pp. 59-90

[BKP84] Barringer, H. \& Kuiper, R. \& Pnueli, A.: Now You May Compose Temporal Logic Specification, in Conference Record of the Sixteenth Annual ACM Symposium on Theory of Computing, 1984, pp. 51-63

[BE91] Best, E. \& Esparza, J.: Model Checking of Persistent Petri Nets, Hildesheimer Informatikberichte 11/91, Universität Hildesheim, Institut für Informatik, 1991, also presented in Computer' Science Logic '91

[CLM89] Clarke, E. M. \& Long, D. E. \& McMillan, K. L.: Compositional Model Checking, in Proceedings of the Fourth IEEE Symposium on Logic in Computer Science, 1989, pp. 353-362

[GL91] Grümberg, O. \& Long, D. E.: Model Checking and Modular Verification, in Baeten, J. C. M. \& Groote, J. F. (eds.): Proceedings of CONCUR'91, the 2nd International Conference on Concurrency Theory, LNCS, vol. 527, Springer-Verlag, 1991, pp. 250-265

[Jos89] Josko, B.: Verifying the Correctness of AADLmodules Using Model Checking, in de Bakker, J.W. \& de Roever, W.-P. \& Rozenberg, G. (eds.): Proceedings of the REX Workshop on Stepwise Refinement of Distributed Systems, Models, Formalisms, Correctness, LNCS, vol. 430, Springer-Verlag, 1989, pp. 386-400

[Lam83] Lamport, L.: What Good is Temporal Logic?, in Proceedings of the IFIP gth World Computer Congress, 1983, pp. 657-668

[LP85] Lichtenstein. O, \& Pnueli, A.: Checking That Finite State Concurrent Programs Satisfy Their Linear Specification, in Conference Record of the Twelfth Annual ACM Symposizm on Principles of Programming Languages, 1985, pp. 97-107

[LPZ85] Lichtenstein. O, \& Pnueli, A. \& Zuck, L.: The Glory of The Past, in Parikh, R. (ed.): Logics of Programs, Proceedings, LNCS, vol. 193, Springer-Verlag, 1985, pp. 196-218

[MP81] Manna, Z.\& Pnueli. A.: Verification of Concurrent Programs: The Temporal Framework, in Boyer, R. S. \& Moore, J. S. (cds.): The Correctness Problem in Computer Science, Academic Press, 1981, pp. 215-273

[MP91] Manna, Z. \& Pnueli. A.: The Temporal Logic of Reactive and Concurrent Systems, vol. 1, Specification, Springer-Verlag, 1991

[Pnu85] Pnueli, A.: In Transition from Global to Modular Temporal Reasoning About Programs, in Apt, K. R. (ed.): Logics and Models of Concurrent Systems, NATO ASI Series, vol. F13, Springer-Verlag, 1985, pp. 123-146 